\title{
Tarifpolitischer Jahresbericht 2011: Höhere Abschlüsse - Konflikte um Tarifstandards
}

\begin{abstract}
Angesichts der günstigen wirtschaftlichen Rahmenbedingungen konnten die Gewerkschaften 2011 eine offensivere Lohn- und Gehaltspolitik betreiben. Die Tarifabschlüsse lagen meist deutlich über denen des Vorjahres. Allerdings fiel der Anstieg der Verbraucherpreise mit rund 2,3 \% gut doppelt so hoch aus wie im Vorjahr. Auf das Jahr gerechnet und unter Berücksichtigung der länger laufenden Abschlüsse aus dem Jahr 2010 konnte deshalb die durchschnittliche Tarifsteigerung von 2,0 \% im Jahr 2011 den Anstieg der Lebenshaltungskosten nicht ausgleichen. Tarifpolitische Neuerungen gab es bei der Übernahme der Ausgebildeten und bei der Arbeitszeitgestaltung. Die branchenbezogenen tariflichen Mindestlöhne nach dem Entsendegesetz wurden ausgeweitet und angehoben.
\end{abstract}

REINHARD BISPINCK, WSI-TARIFARCHIV

\section{Das Tarifjahr 2011 im Überblick}

\subsection{Rahmenbedingungen}

Die Tarifpolitik im Jahr 2011 war geprägt von einer spürbaren Akzentverschiebung: Hatten sich die Gewerkschaften 2010 zumindest in den stark krisenbetroffenen Industriebranchen auf die Sicherung der Beschäftigung konzentriert, stand im vergangenen Jahr die Lohnentwicklung wieder im Vordergrund. Dies war in erster Linie eine Reaktion auf die anhaltende Verbesserung der wirtschaftlichen Rahmenbedingungen. Ein positiver Einfluss ging vor allem von der konjunkturellen Entwicklung aus. Im Jahr 2010 hatte sich die wirtschaftliche Erholung bereits deutlich verstärkt (IMK 2011). Im 4. Quartal betrug die reale Wachstumsrate des Bruttoinlandsprodukts (BIP) 3,8 \%, im 1. Quartal 2011 sogar 5,0 \%. Allerdings schwächte sich die Entwicklung im weiteren Jahresverlauf deutlich ab, sodass im 4. Quartal sogar ein leichtes Schrumpfen des BIP zu verzeichnen war. Auch der Arbeitsmarkt belebte sich: Die Erwerbstätigkeit nahm im Jahr 2011 um 1,3 \% zu, die Zahl der sozialversicherungspflichtig Beschäftigten sogar um 2,4 \%. Die Zahl der registrierten Arbeitslosen sank von 3,34 Mio. im Januar auf 2,78 Mio. im Dezember 2011 (BA 2011). Die wachsenden wirtschaftlichen und politischen Turbulenzen im Zusammenhang mit der ungelösten Eurokrise schlugen auf die tarifpolitische Entwicklung nicht durch, da die deutsche
(Real-)Wirtschaft davon zunächst noch nicht unmittelbar betroffen war. Für die Tarifrunde 2012 zeichnen sich allerdings wieder verschlechterte Rahmenbedingungen ab. Seitens der Politik gab es vielfältige Unterstützung für die gewerkschaftlichen Forderungen nach einer Beteiligung der Beschäftigten an der positiven wirtschaftlichen Entwicklung. Auch eine Reihe von Ökonomen signalisierte einen aus ihrer Sicht gestiegenen Verteilungsspielraum von bis zu $3 \%$ und mehr.

Neben den üblichen Lohn- und Gehaltsrunden wurde in einzelnen Tarifbereichen hart und grundsätzlich um Tarifstandards gestritten, so z. B. in der Druckindustrie und bei den Redakteurinnen und Redakteuren der Tageszeitungen, wo die Arbeitgeber tiefe Einschnitte in bestehende tarifliche Regelungen forderten. Erst nach teils monatelangen, von Arbeitskämpfen begleiteten Verhandlungen konnten Kompromisse gefunden werden, die in beiden Branchen den tarifvertraglichen Regelungsbestand weitgehend erhalten. In anderen Bereichen gelang die Vereinbarung verbesserter qualitativer Tarifregelungen wie z. B. das Equal-PayPrinzip bei Leiharbeit in der Stahlindustrie oder der Tarifvertrag über lebensphasengerechte Arbeitszeitgestaltung in der chemischen Industrie Ostdeutschlands.

Ein Dauerthema war auch im vergangenen Jahr die Regulierung des Niedriglohnsektors und die Festlegung von Mindestlöhnen. So wurden bestehende Mindestlöhne nach dem Entsendegesetz schrittweise angehoben und neue Bereiche in das Gesetz einbezogen. Für den umstrittenen 
Bereich der Leiharbeit wurde eine allgemeinverbindliche Lohnuntergrenze im Arbeitnehmerüberlassungsgesetz festgelegt, die inzwischen auch Rechtskraft erlangt hat. Auch die Festlegung von Mindestlöhnen im Rahmen von Tariftreuebzw. Vergabegesetzen der Bundesländer machte Fortschritte. Die Diskussion um einen allgemeinen branchenübergreifenden gesetzlichen Mindestlohn führte allerdings (noch) nicht zu konkreten politischen Maßnahmen (vgl. Abschnitt 3).

\subsection{Lohn- und Gehaltsforderungen}

Die Tarifforderungen der Gewerkschaften bewegten sich in der Tarifrunde 2011 überwiegend zwischen 5 und $7 \%$. Lediglich im Steinkohlenbergbau fehlte eine bezifferte Forderung, die Gewerkschaft forderte eine „reale Einkommenserhöhung". Am unteren Rand bewegten sich Branchen wie die Textil- und Bekleidungsindustrie (5\%), die Druckindustrie, der Großhandel in Baden-Württemberg (jeweils 5,5\%). Im Mittelfeld lagen Bereiche wie das Bauhauptgewerbe $(5,9 \%)$ sowie Volkswagen und das Versicherungsgewerbe (jeweils $6 \%$ ). Die höchste Tarifforderung für eine größere Industriebranche stellten in dieser Tarifrunde die IG BCE in der chemischen Industrie und die IG Metall in der Stahlindustrie, wo sie jeweils eine Tarifanhebung von $7 \%$ verlangten. Auffällig war, dass in einigen Branchen und Bereichen auch Mindest-, Sockel- und Festbeträge gefordert

\section{ÜBERSICHT 1}

Lohn- und Gehaltsforderungen in der Tarifrunde 2011 in ausgewählten Tarifbereichen

\begin{tabular}{lll}
\hline Tarifbereich & Gewerkschaft & Forderung \\
\hline Bauhauptgewerbe & IG BAU & $5,9 \%$ \\
\hline Chemische Industrie & IG BCE & $7 \%$ \\
\hline Deutsche Telekom AG & ver.di & $6,5 \%$, mind. 170 $€$ \\
\hline Druckindustrie & ver.di & $5,5 \%$ \\
\hline Einzelhandel (NRW) & ver.di & $6,5 \%$, mind. $130 €$ \\
\hline Eisen- und Stahlindustrie & IG Metall & $7 \%$ \\
\hline Energiewirtschaft (NRW - GWE-Bereich) & IG BCE, ver.di & $6,5 \%$ \\
\hline Groß- und Außenhandel (Baden-Württemberg) & ver.di & $5,5 \%$ \\
\hline Holz- und Kunststoffverarbeitung & IG Metall & $5,8 \%$ \\
\hline Kautschukindustrie & IG BCE & $7 \%$ \\
\hline Nahrung-Genuss-Gaststätten & NGG & $5-6 \%$ \\
\hline Öffentlicher Dienst Länder & ver.di & $50 €+3 \%$ ( 5 5 $\%$ ) \\
\hline PrivaterTransport und Verkehr (NRW) & ver.di & $95 €$ \\
\hline Steinkohlenbergbau & IG BCE & reale Einkommenserhöhung \\
\hline Textil- und Bekleidungsindustrie (West) & IG Metall & $5 \%$ \\
\hline Textile Dienstleistungen & IG Metall & $5 \%$ \\
\hline Versicherungsgewerbe & ver.di & $6 \%$, mind. 150 $€$ \\
\hline Volkswagen AG & IG Metall & $6 \%$ \\
\hline & & \\
\hline
\end{tabular}

wurden. Im Transport- und Verkehrsgewerbe NRW forderte ver.di einheitlich $95 €$ für alle Beschäftigten, im Einzelhandel NRW belief sich die Forderung auf 6,5 \%, mindestens $130 € /$ Monat, und bei der Deutschen Telekom AG auf $6,5 \%$, mindestens $170 €$. Im öffentlichen Dienst der Länder sollten die Tabellenvergütungen einheitlich um $50 €$ und anschließend um 3,0 \% erhöht werden (Übersicht 1).

Nach dem Kündigungsterminkalender liefen Ende Dezember 2010 die Tarifverträge im öffentlichen Dienst (Länder) und bei der Deutschen Telekom aus. Ende Januar 2011 folgte die Volkswagen AG. Ende Februar 2011 endete die Laufzeit der Verträge in der chemischen Industrie Nordrhein, Hessen und Rheinland-Pfalz. Die übrigen Regionen waren Ende März und April an der Reihe. Ende März folgten das Bauhauptgewerbe, die Druckindustrie, das Versicherungsgewerbe und einige Regionen des Einzel- und Großhandels und der Süßwarenindustrie. Weitere Bereiche des Einzel- und Großhandels standen Ende April, Mai und Juni auf der Tagesordnung. Ende Oktober lief der Tarifvertrag in der westdeutschen Eisen- und Stahlindustrie aus. Die Tarifverhandlungen in dieser Branche läuteten bereits die Tarifrunde 2012 ein.

Keine Tarifrunde fand im vergangenen Jahr in der Metall- und Elektroindustrie statt. Der Abschluss aus dem Jahr 2010 sah u. a. eine Tarifanhebung von 2,7 \% ab April 2011 vor. Die Laufzeit des Vertrages reicht bis Ende März 2012. Auch im Bankgewerbe, im öffentlichen Dienst (Bund und Gemeinden) und in einigen weiteren Branchen reicht die Laufzeit der Verträge bis ins Jahr 2012. Ein Überblick über die Tarifabschlüsse zeigt folgendes Bild:

Februar: Bei der Volkswagen AG erzielte die IG Metall vor dem Hintergrund hervorragender Geschäftszahlen am 8.2.2011 eine Tariferhöhung von 3,2 \% ab 1.5.2011 bei einer Laufzeit bis Ende Mai 2012. Für die Monate Februar bis April wurde als Pauschale 1,0\% des Jahresentgelts (mind. $500 €)$ gezahlt. In verschiedenen Energieunternehmen (RWE, Vattenfall) wurden Tarifanhebungen um 3,4 \% bei 13 Monaten Laufzeit vereinbart. In der Textil- und Bekleidungsindustrie Westdeutschland sieht der Abschluss vom 21.2.2011 nach zwei Nullmonaten (März und April) eine Pauschalzahlung von $250 €$ für Mai bis September vor, gefolgt von einer Tarifanhebung um 3,6 \% ab 1.10.2011 bis Ende Oktober 2012. Für das Hotel- und Gaststättengewerbe Baden-Württemberg erreichte die Gewerkschaft NGG am 23.2.2011 nach drei Nullmonaten (Januar bis März) eine Tarifsteigerung von 2,9 \% ab 1.4.2011 und weitere 2,4 \% ab 1.7.2012 bei einer Laufzeit bis Ende Juni 2013.

März: Im öffentlichen Dienst der Länder (ohne Hessen und Berlin) erfolgte ein Tarifabschluss am 10.3.2011. Für die Monate Januar bis März wurde eine Pauschale von insgesamt $360 €$ vereinbart. Darauf folgte eine tarifliche Entgelterhöhung von 1,5\% ab dem 1.4.2011 und eine Stufenerhöhung von $1,9 \%$ zuzüglich $17 €$ Sockelbetrag ab dem 1.1.2012. Die Laufzeit reicht bis Ende 2012. Einen vergleichbaren Abschluss erzielte ver.di am 5.4.2011 für das Land Hessen. 
Der Abschluss in der chemischen Industrie erfolgte am 31.3.2011. Er sieht nach einem Nullmonat eine Tarifanhebung von 4,1 \% für jeweils 14 Monate regional unterschiedlich bis 31.5./30.6./31.7.2012 vor. Mit dieser relativ kurzen Laufzeit unterscheidet sich der Abschluss deutlich von den übrigen Abkommen (vgl. Abschnitt 3.2). Die Tarifvertragsparteien haben sich darüber hinaus auf die Fortführung und Ausweitung des Förderprogramms "Start in den Beruf “ geeinigt.

April: Im Bauhauptgewerbe verständigten sich die Tarifparteien erst nach einer Schlichtung am 14.4.2011 auf einen Abschluss: Die Tarifvergütungen werden im Westen und in Berlin nach einem Nullmonat (April) um 3,0 \% ab 1.5.2011 erhöht, ab 1.6.2012 gibt es eine Stufenerhöhung von $2,3 \%$. Im Osten gibt es nach zwei Nullmonaten (April und Mai) etwas stärkere Erhöhungen. Die Tarifverträge laufen insgesamt 24 Monate bis Ende März 2013. Ebenfalls erhöht werden die Mindestlöhne West, Berlin und Ost ab Januar 2012 und 2013 mit einer Laufzeit bis zum 31.12.2013.

Juni: Im Einzelhandel erreichte ver.di einen ersten regionalen Abschluss am 10.6.2011 für den Tarifbezirk BadenWürttemberg: Er sieht nach zwei Nullmonaten Tarifsteigerungen von 3,0 \% ab Juni 2011 und weiteren 2,0 \% ab Juni 2012 bei einer Gesamtlaufzeit von 24 Monaten vor. Außerdem wurden Vereinbarungen zur Überarbeitung von manteltariflichen Regelungen getroffen, die mit dem Allgemeinen Gleichstellungsgesetz nicht vereinbar waren.

Die Tarifparteien in der Druckindustrie einigten sich nach sechs Verhandlungsrunden und intensiven Warnstreiks Ende Juni auf ein Wiederinkraftsetzen des Manteltarifvertrages ohne die von den Arbeitgebern geforderte Verlängerung der Arbeitszeit und die Absenkung der Löhne für Hilfskräfte. Löhne und Gehälter steigen nach einer Pauschalzahlung von $280 €$ insgesamt für April 2011 bis Juli 2012; um 2,0 \% ab August 2012 mit einer Laufzeit bis Ende 2013. Eine zusätzliche Einmalzahlung in Höhe von $150 €$ wird im Juli 2013 gezahlt. Die Gesamtlaufzeit beträgt also 33 (!) Monate.

Juli: Im Versicherungsgewerbe sieht der Abschluss vom 21.7. eine Pauschale von insgesamt $350 €$ für April bis August (450€ für untere Gruppen) und eine Tarifanhebung von $3,0 \%$ ab 1.9.2011 sowie eine Stufenanhebung von weiteren $2,2 \%$ ab 1.10 .2012 bis 31.3.2013 vor.

November: In der Eisen- und Stahlindustrie setzten die Tarifparteien einen Orientierungswert für die Tarifrunde 2012. Der Abschluss vom 22.11.2011 sieht nach einem Nullmonat eine Tarifanhebung von $3,8 \%$ ab 1.12.2011 bis 28.2.2013 vor, die Gesamtlaufzeit beträgt also 16 Monate.

Die Abschlüsse zeigen insgesamt eine große Bandbreite, die den unterschiedlichen ökonomischen Rahmenbedingungen und tarifpolitischen Kräfteverhältnissen in den einzelnen Branchen und Tarifgebieten geschuldet ist (Übersicht 2). Aus Arbeitgebersicht ist gerade dies das hervorstechende und positive Merkmal der Tarifrunde. So kritisierte die Bundesvereinigung der Deutschen Arbeitgeberverbände (BDA) in ihrem Geschäftsbericht 2011 zwar die „überzogenen Tarifforderungen“, mit denen die Gewerkschaften - nicht zuletzt beeinflusst durch Äußerungen aus Teilen der Politik - in die Tarifrunden gestartet seien, lobten aber die „differenzierten Tarifabschlüsse“, die bewiesen, „dass die Zeiten der Geleitzüge in der Tarifpolitik endgültig vorbei“ seien (BDA 2011).

\section{Tarifentwicklung - Daten zur Tarifstatistik}

\subsection{Lohn und Gehalt}

Die DGB-Gewerkschaften schlossen 2011 in ganz Deutschland Lohn- und Gehaltstarifverträge für 9,2 Mio. Beschäftigte ab, davon für rund 7,9 Mio. in den alten und 1,3 Mio. in den neuen Bundesländern. Das entspricht rund $49 \%$ der von Tarifverträgen erfassten Beschäftigten. Für weitere 7,4 Mio. Beschäftigte traten Stufenerhöhungen in Kraft, die bereits 2010 oder früher vereinbart worden waren. Bei rund 2,1 Mio. Beschäftigten liefen 2010 oder früher die Vergütungstarifverträge aus, aber es kam bis zum Jahresende (noch) nicht zu Neuabschlüssen bzw. laufende Tarifverträge sehen für 2011 keine Tarifanhebung vor.

\subsubsection{Abschlussrate}

Die tarifliche Abschlussrate belief sich gesamtwirtschaftlich im Durchschnitt auf 4,8 \% (2010: 3,2\%), in Westdeutschland betrug die Rate 4,7\%, in Ostdeutschland 5,4\%. Die Abschlussrate schließt alle, ggf. auch 2012 und später in Kraft tretenden tabellenwirksamen Erhöhungen ein. Nicht berücksichtigt werden Pauschalzahlungen und zusätzliche Einmalzahlungen, die sich nicht dauerhaft in den Tariftabellen niederschlagen. Die Spannweite der durchschnittlichen Gesamtabschlussraten reicht von 3,4\% im Verbrauchsgütergewerbe, über 4,0 \% im Bereich Gebietskörperschaften, Sozialversicherung sowie Energie- und Wasserversorgung, Bergbau, 4,3 \% im Investitionsgütergewerbe, 5,3 \% im Handel, 5,5 \% im Baugewerbe bis zu 5,8 \% im Bereich Gartenbau, Land- und Forstwirtschaft.

Diese Gesamtabschlussraten sind nur von begrenzter Aussagekraft, weil sie sich immer auf die gesamte, je nach Tarifbereich sehr unterschiedlich lange Laufzeit der Tarifabkommen beziehen. Berücksichtigt man lediglich die im Jahr 2011 abgeschlossenen und auch in Kraft getretenen Tariferhöhungen, ergibt sich eine Abschlussrate von 3,0 \% (West: 3,0 \%; Ost: 2,9\%). Differenziert man diese Größe nach Wirtschaftsbereichen, dann ergibt sich für 2011 eine Streuung zwischen 1,5 \% im Bereich Gebietskörperschaften/Sozialversicherung und 3,8 \% im Grundstoff- und Produktionsgütergewerbe sowie im Verbrauchsgütergewerbe. In Rechnung zu stellen ist, dass die Erhöhungen zu verschiedenen Zeitpunkten im Jahresverlauf wirksam wurden 
ÜBERSICHT 2

\section{Ausgewählte Lohn- und Gehaltsabschlüsse West und Ost für 2011}

\begin{tabular}{|c|c|c|c|c|}
\hline Abschluss & Tarifbereich & 2011 & 2012 & Laufzeit in Mon. \\
\hline $\begin{array}{l}09.12 .2010 \\
25.01 .2011\end{array}$ & Deutsche Bahn AG & 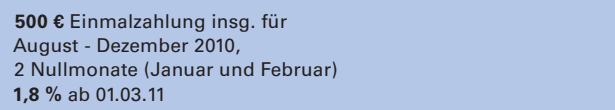 & $\begin{array}{l}2,0 \% \text { ab } 01.01 .12 \\
\text { bis } 31.12 .12\end{array}$ & 29 \\
\hline 31.01 .2011 & Privater Transport und Verkehr NRW & $\begin{array}{l}\text { nach } 4 \text { Nullmonaten (Dezember } 2010 \text { - März 2011) } \\
\mathbf{3 , 1} \% \text { ab 01.04.11 }\end{array}$ & $\begin{array}{l}1,7 \% \text { ab } 01.03 .12 \\
\text { bis } 28.02 .13\end{array}$ & 27 \\
\hline 08.02 .2011 & Volkswagen AG & $\begin{array}{l}\mathbf{1 , 0} \% \text { des Jahresentgelts, mind. } 500 € \\
\text { als Pauschale insg. für Februar - April } \\
\mathbf{3 , 2} \% \text { ab } 01.05 .11 \text { bis } 31.05 .12\end{array}$ & & 16 \\
\hline 21.02 .2011 & Steinkohlenbergbau & $\begin{array}{l}600 € \text { Pauschale insg. für Januar - März } \\
\mathbf{3 , 3} \% \text { ab } 01.04 .11 \text { bis } 31.12 .12\end{array}$ & & 24 \\
\hline 21.02.2011 & Textil- und Bekleidungsindustrie West & $\begin{array}{l}\text { nach } 2 \text { Nullmonaten (März und April) } \\
250 € \text { Pauschale insg. für Mai - September } \\
\mathbf{3 , 6} \% \text { ab } 01.10 .11 \text { bis } 31.10 .12\end{array}$ & & 20 \\
\hline 23.02.2011 & $\begin{array}{l}\text { Hotels und Gaststätten } \\
\text { Baden-Württemberg }\end{array}$ & $\begin{array}{l}\text { nach } 3 \text { Nullmonaten (Januar - März) } \\
2,9 \% \text { ab 01.04.11 }\end{array}$ & $\begin{array}{l}2,4 \% \text { ab } 01.07 .12 \\
\text { bis } 30.06 .13\end{array}$ & 30 \\
\hline 10.03.2011 & Öffentlicher Dienst Länder & $\begin{array}{l}360 € \text { Pauschale insg. für Januar - März } \\
1,5 \% \text { ab } 01.04 .11\end{array}$ & $\begin{array}{l}\text { 1,9\% plus } 17 € \text { ab } 01.01 .12 \\
\text { bis } 31.12 .12\end{array}$ & 24 \\
\hline 24.03.2011 & Textilindustrie Ost & $\begin{array}{l}\text { nach } 2 \text { Nullmonaten (April und Mai) } \\
2,5 \% \text { ab } 01.06 .11\end{array}$ & $\begin{array}{l}2,3 \% \text { ab } 01.04 .12 \\
\text { bis } 31.03 .13\end{array}$ & 24 \\
\hline 25.03.2011 & $\begin{array}{l}\text { Deutsche Telekom AG, } \\
\text { Deutsche Telekom Servicegesellschaften }\end{array}$ & $\begin{array}{l}\text { nach } 3 \text { Nullmonaten (Januar - März) (für bestimmte AN-Gruppen } \\
\text { statt Nullmonate } 2,0 \% \text { ab 01.01.11) } \\
\mathbf{3 , 1 5} \% \text { ab } 01.04 .11 \\
\text { Mindesterhöhung von } 75 € / \text { Mon. } \\
\text { bis } 31.01 .12\end{array}$ & & 13 \\
\hline 28.03.2011 & Textiles Reinigungsgewerbe & $\begin{array}{l}\text { nach } 2 \text { Nullmonaten (April und Mai) } \\
2,7 \% \text { ab 01.06.11, Ost: Erhöhung um Steigerungsbeträge West }\end{array}$ & $\begin{array}{l}2,3 \% \text { ab } 01.06 .12 \text {, Ost: Erhöhung um } \\
\text { Steigerungsbeträge West } \\
\text { bis } 31.05 .13\end{array}$ & 26 \\
\hline 31.03.2011 & Chemische Industrie & $\begin{array}{l}\text { nach } 1 \text { Nullmonat } \\
\mathbf{4 , 1} \% \text { für jew. } 14 \text { Monate regional unterschiedlich bis } \\
31.05 . / 30.06 . / 31.07 .12\end{array}$ & & 15 \\
\hline 14.04 .2011 & Bauhauptgewerbe & $\begin{array}{l}\text { nach } 1 \text { bzw. } 2 \text { Nullmonaten (April und Mai) } \\
\mathbf{3 , 0} \% \text { ab 01.05.11 (West) } \\
\mathbf{3 , 4} \% \text { ab 01.06.11 (Ost) }\end{array}$ & $\begin{array}{l}2,3 \% \text { ab } 01.06 .12 \text { (West) } \\
2,9 \% \text { ab } 01.08 .12 \text { (Ost) } \\
\text { bis } 31.03 .13 \\
\text { Anhebungen des Mindestlohns }\end{array}$ & 24 \\
\hline 18.04.2011 & Süßwarenindustrie & $\begin{array}{l}\text { nach } 1 \text { Nullmonat (regional unterschiedlich) } \\
\mathbf{3 , 0} \% \text { ab 05/06/07/08/09.11 bzw. } 02.12\end{array}$ & $\begin{array}{l}2,8 \% \text { ab 05/06/07/08/ } \\
09.12 \text { bzw. } 02.13 \text { für jew. } 12 \text { Mon. }\end{array}$ & 25 \\
\hline 20.05 .2011 & $\begin{array}{l}\text { Groß- und Außenhandel } \\
\text { Baden-Württemberg }\end{array}$ & $\begin{array}{l}\text { nach } 1 \text { Nullmonat (April) } \\
\mathbf{3 , 0} \% \text { ab } 01.05 .11\end{array}$ & $\begin{array}{l}2,4 \% \text { ab } 01.05 .12 \\
\text { bis } 31.03 .13\end{array}$ & 24 \\
\hline 10.06 .2011 & Einzelhandel Baden-Württemberg & $\begin{array}{l}\text { nach } 2 \text { Nullmonaten (April und Mai) } \\
\mathbf{3 , 0} \% \text { ab 01.06.11 }\end{array}$ & $\begin{array}{l}\mathbf{2 , 0} \% \text { ab } 01.06 .12 \\
\text { bis } 31.03 .13 \\
\mathbf{5 0} € \text { zus. Einmalzahlung } 04.12\end{array}$ & 24 \\
\hline 21.06 .2011 & $\begin{array}{l}\text { Holz und Kunststoff verarbeitende } \\
\text { Industrie Westfalen-Lippe }\end{array}$ & $\begin{array}{l}360 € \text { Pauschale insg. für Mai-Oktober } \\
4,0 \% \text { ab } 01.11 .11 \text { bis } 31.12 .12\end{array}$ & & 20 \\
\hline 29.06.2011 & Druckindustrie & $280 €$ Pauschale insg. für April 2011 - Juli 2012 & $\begin{array}{l}2,0 \% \text { ab } 01.08 .12 \text { bis } 31.12 .13 \\
150 € \text { zus. Einmalzahlung } 07.13\end{array}$ & 33 \\
\hline 11.07 .2011 & Energiewirtschaft NRW (GWE) & $3,3 \%$ ab 01.07 .11 bis 31.08 .12 & & 13 \\
\hline 21.07.2011 & Versicherungsgewerbe & $\begin{array}{l}350 € \text { Pauschale insg. für April - August } \\
\text { (450€ für untere Gruppen) } \\
\mathbf{3 , 0} \% \text { ab 01.09.11 }\end{array}$ & $2,2 \%$ ab 01.10 .12 bis 31.03 .13 & 24 \\
\hline 22.11.2011 & $\begin{array}{l}\text { Eisen- u. Stahlindustrie NRW, } \\
\text { Niedersachsen und Bremen }\end{array}$ & $\begin{array}{l}\text { nach } 1 \text { Nullmonat (November) } \\
\mathbf{3 , 8} \% \text { ab } 01.12 .11 \text { bis } 28.02 .13\end{array}$ & & 16 \\
\hline 29.11.2011 & Tarifgruppe RWE & $2,7 \%$ ab 01.12 .11 bis 31.12 .12 & & 13 \\
\hline 07.12 .2011 & Papier erzeugende Industrie & $70 €$ Pauschale für Dezember & $\begin{array}{l}\mathbf{3}, \mathbf{0} \% \text { ab } 01.01 .12 \\
\mathbf{1 , 6} \% \text { ab } 01.01 .13 \text { bis } 31.05 .13\end{array}$ & 18 \\
\hline
\end{tabular}


und damit auch die Auswirkungen auf die Tarifvergütungen des Jahres 2011 unterschiedlich waren.

Im Jahr 2011 spielten „Nullmonate“ bei den Tarifabschlüssen eine deutlich geringere Rolle als noch im Vorjahr. Zwar gab es für rund 8,1 Mio. (2010: 6,9 Mio.) Tarifabschlüsse mit verzögerter Anpassung der Lohn- und Gehaltserhöhungen, was rund $88 \%$ der von Neuabschlüssen begünstigten Beschäftigten entspricht. Aber die Zahl der Verzögerungsmonate blieb deutlich unter dem Vorjahr. Rund 78 \% der genannten Beschäftigten mussten zwischen einem Monat und fünf Monate auf die reguläre Tariferhöhung warten, für $10 \%$ vergingen sechs und mehr Monate bis zur ersten Tarifsteigerung. Für 2,2 Mio., das sind $27 \%$ der davon betroffenen Beschäftigten, vereinbarten die Gewerkschaften als Ausgleich Pauschalzahlungen, die durchschnittlich $82 €$ (West: $81 €$; Ost: $86 €$ ) im Monat betrugen.

\subsubsection{Laufzeiten}

Der seit rund zehn Jahren zu beobachtende Trend zu fast zweijährigen Laufzeiten hat sich im vergangenen Jahr fortgesetzt. Die Laufzeit der Vergütungstarifverträge im Jahr 2011 beträgt durchschnittlich 22,8 Monate (2010: 24,3 Monate). Für rund 1,5 Mio. Beschäftigte (16,0 \%) laufen die Abkommen zwischen elf und 15 Monaten, für rund 0,4 Mio. (4,3\%) 16 bis 19 Monate und für 7,3 Mio. (79,7 \%) 20 Monate und länger. In den neuen Bundesländern laufen die Tarifverträge im Schnitt gut einen Monat länger als in den alten (Tabelle 1).

\subsubsection{Jahresbezogene Tarifsteigerung}

Bei der Berechnung der auf das Kalenderjahr bezogenen Steigerung der tariflichen Grundlöhne und -gehälter werden im Unterschied zur tariflichen Abschlussrate die Auswirkungen aus der unterschiedlichen Lage und Laufzeit der Tarifabkommen berücksichtigt. Auch werden ggf. im Berichtsjahr wirksam werdende Abschlüsse aus den Vorjahren sowie zusätzliche Einmalzahlungen und Pauschalzahlungen als Ausgleich für Abschlussverzögerungen mit einbezogen. Die jahresbezogene Tarifsteigerung setzt die durchschnittliche tarifliche Grundvergütung des gesamten Jahres 2011 zum Vorjahr in Bezug und erfasst insgesamt 16,8 Mio. abhängig Beschäftigte.

Diese kalenderjährliche Steigerung der Tarifverdienste 2011 gegenüber 2010 betrug für ganz Deutschland 2,0\% (2010: 1,8 \%) (Tabelle 2). Am höchsten fiel die jahresbezogene Tarifsteigerung mit 2,8 \% im Bereich Private Dienstleistungen, Organisationen ohne Erwerbszweck aus, gefolgt vom Nahrungs- und Genussmittelgewerbe und dem Baugewerbe mit 2,3\% und dem Grundstoff- und Produktionsgütergewerbe mit 2,2 \%. Genau im Durchschnitt lagen mit 2,0 \% das Verbrauchsgütergewerbe und der Handel. Unterdurchschnittlich fielen die Tarifsteigerungen mit 1,8 \% im Investitionsgütergewerbe sowie im Bereich Gebietskörperschaften, Sozialversicherung aus. Darunter lagen die Berei- che Gartenbau, Land- und Forstwirtschaft mit 1,6\% und die Finanzdienstleistungen mit 1,1\%. In Ostdeutschland lag die kalenderjährliche Erhöhung mit 2,2 \% etwas höher als in Westdeutschland mit 2,0\%.

Leicht negativ beeinflusst wird die jahresbezogene Tarifsteigerung 2011 durch die länger laufenden Abschlüsse aus dem Jahr 2010. Die daraus resultierende Tarifanhebung für 2011 beläuft sich auf 1,9\%, die Neuabschlüsse des Jahres 2011 ergeben dagegen 2,1\%. Im Mittel ergeben sich dann die genannten $2,0 \%$.

In Tarifbereichen mit rund 281.000 Beschäftigten liefen Vergütungstarifverträge im Jahr 2011 aus, ohne dass bis zum Jahresende neue Abschlüsse getätigt wurden. Bezieht man diese Bereiche mit ein, ergibt sich wegen der geringen

\section{TABELLE 1}

\section{Laufzeit der Tarifverträge}

Angaben in Monaten

\begin{tabular}{ll|l|l|l|l|l|l|l|l|l|l}
\hline & $\mathbf{2 0 0 1}$ & $\mathbf{2 0 0 2}$ & $\mathbf{2 0 0 3}$ & $\mathbf{2 0 0 4}$ & $\mathbf{2 0 0 5}$ & $\mathbf{2 0 0 6}$ & $\mathbf{2 0 0 7}$ & $\mathbf{2 0 0 8}$ & $\mathbf{2 0 0 9}$ & $\mathbf{2 0 1 0}$ & $\mathbf{2 0 1 1}$ \\
\hline Gesamt & 14,5 & $\mathbf{1 8 , 3}$ & 20,5 & 21,8 & 25,7 & 22,1 & 22,2 & 22,4 & 24,1 & 24,3 & 22,8 \\
\hline West & 14,1 & 18,1 & 20,4 & 21,8 & 25,2 & 21,6 & 22,2 & 22,2 & 24,3 & 23,6 & 22,6 \\
\hline Ost & 16,4 & 19,7 & 21,0 & 22,0 & 28,4 & 24,7 & 21,9 & 23,4 & 23,2 & 28,3 & 23,9 \\
\hline
\end{tabular}

Quelle:WSI-Tarifarchiv, Stand: 31.12.2011.

TABELLE 2

\section{Tarifsteigerung 2011}

Angaben in Prozent ${ }^{1}$

\begin{tabular}{l|l|l|l}
\hline Wirtschaftsbereich & Ost & West & Gesamt \\
\hline Gartenbau, Land- und Forstwirtschaft & 1,8 & 1,6 & 1,6 \\
\hline Energie- und Wasserversorgung, Bergbau & 0,1 & 2,4 & 2,1 \\
\hline Grundstoff- und Produktionsgütergewerbe & 2,4 & 2,2 & 2,2 \\
\hline Investitionsgütergewerbe & 1,6 & 1,8 & 1,8 \\
\hline Verbrauchsgütergewerbe & 2,0 & 2,0 & 2,0 \\
\hline Nahrungs- und Genussmittelgewerbe & 3,0 & 2,2 & 2,3 \\
\hline Baugewerbe & 2,3 & 2,3 & 2,3 \\
\hline Handel & 1,9 & 2,0 & 2,0 \\
\hline Verkehr und Nachrichtenübermittlung & 0,9 & 1,6 & 1,6 \\
\hline Kreditinstitute, Versicherungsgewerbe & 1,1 & 1,1 & 1,1 \\
\hline Priv. Dienstleistungen, Organ. o. Erwerbszweck & 3,6 & 2,6 & 2,8 \\
\hline Gebietskörperschaften, Sozialversicherung & 1,9 & 1,8 & 1,8 \\
\hline Gesamte Wirtschaft & 2,2 & 2,0 & 2,0 \\
\hline
\end{tabular}

1 Jahresbezogene Erhöhung der tariflichen Grundvergütung 2011 gegenüber 2010. Quelle: WSI-Tarifarchiv, Stand: 31.12.2011 
Zahl keine Auswirkung auf die jahresbezogene Tarifsteigerung.

In Tarifbereichen mit weiteren 1,7 Mio. Beschäftigten sind die Tarifverträge bereits 2010 oder in den Jahren zuvor ohne nachfolgende Abschlüsse ausgelaufen. Berücksichtigt man auch diese Bereiche, dann sinkt die jahresbezogene Tarifsteigerung für alle von Tarifverträgen erfassten Beschäftigten für 2011 insgesamt auf 1,8\%.

Die durchschnittliche jahresbezogene Tarifsteigerung 2011 von 2,0 \% liegt unter dem Anstieg der Lebenshaltungskosten von 2,3\%. Real sanken die tariflichen Grundvergütungen im gesamtwirtschaftlichen Durchschnitt um 0,3\%.

\subsubsection{Effektivverdienstentwicklung}

Die Effektivverdienstentwicklung verzeichnet im vergangenen Jahr gegenüber dem Krisenjahr 2010 einen Erholungsprozess. Die Summe der Bruttolöhne und -gehälter ist 2011 um $4,8 \%$ gestiegen. Je Arbeitnehmerin bzw. Arbeitnehmer ergibt sich auf Monatsbasis - nominal - ein Anstieg um 3,4 \%, auf Stundenbasis dagegen um 2,8 \% (Tabelle 3). Daraus ergibt sich, dass die Bruttoverdienste 2011 erneut real (preisbereinigt) gestiegen sind, und zwar um 1,1 \% auf Monatsbasis bzw. 0,5 \% auf Stundenbasis.

Für 2011 ist daher, wie bereits in schwächerem Umfang im Vorjahr, eine positive Lohndrift festzustellen. Die Ursache liegt zum einen im Arbeitszeiteffekt (rückläufige Kurzarbeit und vermehrte Überstunden), zum anderen in nicht tariflich geregelten Bonuszahlungen in bestimmten Branchen und Betrieben.

Was den kostenneutralen Verteilungsspielraum aus Preisund Produktivitätsentwicklung in Höhe von 3,5 \% betrifft, wurde er im Jahre 2011 durch die Tarifentwicklung mit durchschnittlich 2,0 \% bei Weitem nicht ausgeschöpft, die Effektivlohnentwicklung bleibt ebenfalls auf Monatsbasis mit 3,4 \% leicht, auf Stundenbasis mit 2,8 \% deutlich darunter (Tabelle 3).

Ein Blick auf die funktionelle Einkommensverteilung

\section{TABELLE 3}

\section{Wirtschafts- und Verteilungsdaten 2011}

Veränderung gegenüber dem Vorjahr in Prozent

\begin{tabular}{lc}
\hline Verbraucherpreise & $+2,3$ \\
\hline Arbeitsproduktivität/Stunde & $+1,2$ \\
\hline Neutraler Verteilungsspielraum (Preise + Produktivität) & $+3,5$ \\
\hline Tarifentgelte & $+\mathbf{2 , 0}$ \\
\hline Bruttoverdienste/Arbeitnehmer & $+3,4$ \\
\hline Bruttoverdienste/Stunde & $+2,8$ \\
\hline Lohnstückkosten/Stunde & $+1,5$ \\
\hline Unternehmens- und Vermögenseinkommen & $+1,5$ \\
\hline
\end{tabular}

Quelle: Statistisches Bundesamt, WSI-Tarifarchiv zeigt, dass im Jahr 2011 die Unternehmens- und Vermögenseinkommen mit einem Plus von 1,5 \% schwächer gewachsen sind als die Arbeitnehmerentgelte mit 4,5\%.

\subsubsection{Lohnangleichung Ost/West}

Der Stand der tariflichen Lohnangleichung an das Westniveau kann an der Entwicklung der tariflichen Grundvergütung festgemacht werden. Für den Stichtag 31.12.2011 ergibt sich dabei folgendes Bild: Auf Basis von rund 50 Tarifbereichen/-branchen mit 1,63 Mio. erfassten Beschäftigten errechnet sich ein durchschnittliches Tarifniveau von 96,5\% (Tabelle 4). Damit ergibt sich gesamtwirtschaftlich praktisch eine unveränderte Situation gegenüber dem Vorjahr.

\subsubsection{Ausbildungsvergütungen}

Die Steigerung der tariflichen Ausbildungsvergütungen ist im vergangenen Jahr deutlich kräftiger ausgefallen als 2010. Nach Berechnungen des Bundesinstituts für Berufsbildung (BIBB) ergibt sich ein Anstieg von 3,2 \% (West: 2,9\%; Ost: $4,9 \%$ ), der exakt der Steigerung der tariflichen Grundvergütungen entspricht (BIBB 2012). Je nach Tarifbereich verbergen sich hinter diesen Durchschnittszahlen große Unterschiede: Gemessen an der Ausbildungsvergütung im dritten Ausbildungsjahr variierte die Steigerung in 26 ausgewählten Tarifbereichen zwischen 1,1 \% und 12,4 \%. In fünf Tarifbereichen sind die Ausbildungsvergütungen im vergangenen Jahr regional oder bundesweit gar nicht angehoben worden.

\subsection{Arbeitszeit}

Die Eckdaten der tariflichen Wochenarbeitszeit sind im Laufe des vergangenen Jahres weitgehend gleich geblieben. Die tarifliche Wochenarbeitszeit betrug Ende 2011 im gesamtwirtschaftlichen Durchschnitt in ganz Deutschland 37,7 Stunden (West: 37,5; Ost: 38,8 Stunden) (Übersicht 3). Angesichts der zahlreichen arbeitszeitbezogenen Öffnungsklauseln und Flexi-Bestimmungen (u. a. mit Kontenregelungen und langen Ausgleichszeiträumen) müssen die gesamtwirtschaftlichen und branchenbezogenen Zahlen zur tariflichen Arbeitszeitdauer eher als Referenzgrößen denn als Beschreibung der tatsächlichen Arbeitszeitstandards angesehen werden.

Die tarifliche Urlaubsdauer (Endstufe) beträgt im gesamtdeutschen Durchschnitt unverändert 30,0 Tage (West: 30,1; Ost: 29,5 Tage).

Errechnet man auf Basis dieser und weiterer Einzelkomponenten die tarifliche Jahresarbeitszeit, so ergibt sich ein gesamtdeutscher Durchschnitt von 1.659,5 Stunden, für Westdeutschland 1.649,0 und für Ostdeutschland 1.713,6 Stunden. 


\section{Niedriglöhne und Mindestlöhne - neue Entwicklungen}

Nach wie vor arbeitet ein hoher Anteil der Beschäftigten im Niedriglohnsektor (Tabelle 5). Das belegen seit Jahren Auswertungen verschiedener Datensätze wie etwa des Soziooekonomischen Panels (SOEP) (z. B. Kalina/Weinkopf 2010) und der Beschäftigtenstatistik der Bundesagentur für Arbeit (BA) (z. B. Schulten 2011). Die BA-Daten zeigen, dass im Jahr 2010 knapp $21 \%$ der Vollzeitbeschäftigten mit ihrem Verdienst unterhalb der Niedriglohnschwelle von Zweidrittel des Medianlohns lagen.

Angesichts der Persistenz des Niedriglohnproblems verwundert es nicht, dass die Thematik auch im vergangenen Jahr Gegenstand intensiver tarif- und sozialpolitischer Diskussion und Auseinandersetzung auf verschiedenen Ebenen war.

\subsection{Tarifliche Niedriglöhne}

Eine Untersuchung des WSI-Tarifarchivs von rund 4.700 tariflichen Vergütungsgruppen im Frühjahr 2010 hatte ergeben, dass rund $16 \%$ der Tarifgruppen unterhalb des von den Gewerkschaften geforderten Mindestlohns von 8,50€ lagen. Eine erneute Analyse auf dem Stand von September 2011 ergab eine erkennbare Verbesserung: Nur noch $13 \%$ der Tarifgruppen entsprechen nicht dem selbst gesetzten Maßstab (Abbildung 1). Insbesondere in den ausgeprägten Niedriglohnbranchen Bewachungsgewerbe, Erwerbsgartenbau, Friseurhandwerk, Gebäudereinigerhandwerk und Hotel- und Gaststättengewerbe konnte die Zahl der Tarifgruppen mit Vergütungen unter 7,50€ gesenkt werden (Bispinck/WSI-Tarifarchiv 2011).

\subsection{Mindestlöhne nach dem Entsendegesetz}

Die Gewerkschaften bemühten sich zum einen, die bestehenden tariflichen Mindestlöhne nach dem Arbeitnehmerentsendegesetz anzuheben bzw. die Rechtsverordnung für neu abgeschlossene Mindestlöhne durchzusetzen, und zum anderen, neue Branchen in den Geltungsbereich des Entsendegesetzes zu bringen. Die Bilanz dieser Aktivitäten fällt gemischt aus:

\subsubsection{Neue Mindestlöhne}

- Forstliche Dienstleistungsunternehmen West und Ost: Bereits am 12.10.2010 hatte die IG BAU für diesen Tarifbereich einen Mindestlohn von 10,52 € vereinbart, der am 1.12.2011 auf $10,78 €$ steigen sollte. Eine Aufnahme der Branche in den Geltungsbereich des Arbeitnehmer-Entsendegesetzes (AEntG) ist bislang nicht erfolgt.

- Steinmetz- und Steinbildhauerhandwerk: Am 8.3./8.12.2011 (West/Ost) wurde erstmalig ein Mindestlohn vereinbart in Höhe von 11,00 € für West sowie Berlin-Ost und 9,75 € für
TABELLE 4

\section{Tarifniveau Ost/West}

Angaben in Prozent

\begin{tabular}{|c|c|c|c|c|c|c|c|c|c|c|}
\hline 2001 & 2002 & 2003 & 2004 & 2005 & 2006 & 2007 & 2008 & 2009 & 2010 & 2011 \\
\hline 92,3 & 92,8 & 93,4 & 94,0 & 94,6 & 95,1 & 95,2 & 96,8 & 96,1 & 96,6 & 96,5 \\
\hline
\end{tabular}

Quelle: WSI-Tarifarchiv, Stand: 31.12.2011.

ÜBERSICHT 3

Tarifliche Arbeitszeitregelungen 2011

\begin{tabular}{l|r|r|r}
\hline \multicolumn{1}{l}{ Tarifregelung } & West & Gesamt \\
\hline Wochenarbeitszeit (Std.) & 38,8 & 37,5 & 37,7 \\
\hline Anteil der Beschäftigten (in \%) mit: & 7,1 & 24,7 & 21,8 \\
\hline bis zu 35 Std. & 5,3 & 9,0 & 8,3 \\
\hline $36-37$ Std. & 30,8 & 36,7 & 35,7 \\
\hline $37,5-38,5$ Std. & 56,4 & 29,2 & 33,6 \\
\hline $39-40$ und mehr Std. & 29,5 & 30,1 & 30,0 \\
\hline Urlaub (Arbeitstage) ${ }^{1}$ & $1.713,6$ & $1.649,0$ & $1.659,5$ \\
\hline Jahresarbeitszeit (Std.) & & & \\
\hline
\end{tabular}

1 Endstufe.

Quelle: WSI-Tarifarchiv, Stand: 31.12.2011.

TABELLE 5

Anteil der Vollzeitbeschäftigten im Niedriglohnbereich

Angaben in Prozent

\begin{tabular}{|c|c|c|c|c|c|c|c|c|c|c|c|}
\hline 1999 & 2000 & 2001 & 2002 & 2003 & 2004 & 2005 & 2006 & 2007 & 2008 & 2009 & 2010 \\
\hline 16,9 & 17,3 & 17,6 & 17,9 & 18,4 & 19,0 & 19,5 & 20,1 & 20,4 & 20,5 & 20,4 & 20,9 \\
\hline
\end{tabular}

Getrennte Niedriglohnschwelle von zwei Drittel des Medianlohnes für West- und Ostdeutschland Quelle: Bundesagentur für Arbeit, Beschäftigtenstatistik.

Ost ohne Berlin-Ost. Die Allgemeinverbindlichkeit auf Grundlage des AEntG wurde beantragt, ist aber noch nicht erfolgt.

- Gerüstbauerhandwerk: Erstmalig wurde für diese Branche ein Tarifvertrag über einen einheitlichen Mindestlohn von 9,50€ und eine Anhebung auf 10,00 $€$ ab November 2012 vereinbart. Er wurde noch nicht für allgemeinverbindlich erklärt.

\subsubsection{Anhebung von bestehenden Mindestlöhnen}

- Sicherheitsdienstleistungen Bundesgebiet West und Ost: Am 11.2.2011 vereinbarte ver.di einen überarbeiteten Tarifvertrag zum Mindestlohn für Beschäftigte im Objektschutz-/Separatwachdienst, der regional unterschied- 
liche Mindestlöhne von 6,53 bis 8,60€ vorsieht, die bis Anfang 2013 stufenweise auf 7,50 bis 8,90€ steigen sollen Die Rechtsverordnung trat am 1.6.2011 in Kraft.

- Bauhauptgewerbe: Der Tarifabschluss vom 14.4.2011 sieht eine Anhebung des zweigeteilten Mindestlohnes West von 11,00/13,00 € in zwei Schritten Anfang 2012 und 2013 vor Der Mindestlohn Ost von 9,75€ steigt ebenfalls 2012 und 2013 (siehe Abschnitt 3.3). Die Rechtsverordnung erfolgte im Oktober 2011.

- Abfallwirtschaft: Der bestehende Mindestlohntarifvertrag wurde am 16.6.2011 verlängert mit einer Erhöhung von 8,24 auf 8,33 € ab 1.9.2011 bei einer Laufzeit bis zum 31.3.2012. Der Mindestlohn ist allgemeinverbindlich.

- Bergbauspezialgesellschaften: Mit Abschluss vom 22.3.2011 wurde der Mindestlohn von 11,17 auf 11,53€ bzw. von 12,41 auf $12,81 €$ erhöht. Auch hier ist die Rechtsverordnung erfolgt.

- Gebäudereinigerhandwerk: Mit dem Tarifabschluss vom 24.8.2011 wurde der Mindestlohn für West und Berlin-Ost/ Ost in der Innen- und Unterhaltsreinigung auf 8,82/7,33€ und in der Glas- und Fassadenreinigung unverändert auf $11,33 / 8,88 €$ festgelegt. Eine weitere Anhebung folgt Anfang 2013. Die Regelungen sind inzwischen rechtsgültig.

Insgesamt bestehen Anfang 2012 in zehn Branchen gültige Mindestlöhne nach dem AEntG, für weitere drei Branchen sind vorliegende Mindestlöhne noch nicht allgemeinverbindlich erklärt.

\subsection{Lohnuntergrenze nach dem Arbeitnehmerüberlassungsgesetz}

Die DGB-Tarifgemeinschaft Zeitarbeit und die beiden größten Arbeitgeberverbände der Branche, der Bundesverband Zeitarbeit und der Interessenverband Deutscher Zeitarbeitsunternehmen, hatten sich bereits im Mai 2006 auf einen Mindestlohn von 7,00€ im Westen und 6,10€ im Osten geeinigt. Im Februar 2008 hatten sie erfolglos die Aufnahme in das Entsendegesetz beantragt, weil sich die Regierungsparteien der damaligen großen Koalition aus CDU/CSU und SPD nicht einigen konnten. Die schwarz-gelbe Koalition aus CDU/CSU und FDP, die im Herbst 2009 die Regierung übernahm, lehnte eine Aufnahme in das Entsendegesetz ab und fügte nach langen Kontroversen im April 2011 schließlich die Möglichkeit zur Festlegung einer Lohnuntergrenze in das Arbeitnehmerüberlassungsgesetz (AÜG) ein.

Im Dezember 2011 gab dann der Tarifausschuss beim Bundesarbeitsminister seine Zustimmung und kurz darauf konnte - fünf Jahre nach der ersten tariflichen Vereinbarung - ein Mindestlohn für die Branche rechtsverbindlich gemacht werden. Die Mindestentgelte betragen 7,89€ im Westen und 7,01€ im Osten. Ab dem 1.112012 sollen sie auf 8,19€ im Westen und 7,50€ im Osten steigen.

Ohne Ergebnis blieb die Forderung nach gleicher Entlohnung von Leiharbeitnehmern bei gleichwertiger Arbeit (Equal Pay-Prinzip). Der Druck auf die Politik, in dieser
ABB.

\section{Verteilung tariflicher Vergütungsgruppen}

Angaben in Prozent

- März 2010 n September 2011

bis $7,49 €$

7

7,50 bis $8,49 €$

17

6

8,50 bis $8,99 €$

- 3

9,00 bis $9,99 €$

9

8

$10,00 €$ und mehr

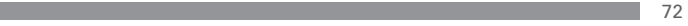

Stand: September 2011

Quelle:WSI-Tarifarchiv.

Frage tätig zu werden, nahm zu, die Gewerkschaften forderten eine Anwendung ab dem ersten Tag des Einsatzes von Leiharbeitnehmern und eine entsprechende gesetzliche Regelung. Die Bundesregierung lehnte entsprechende Maßnahmen ab und forderte stattdessen die Tarifvertragsparteien auf, einen gemeinsamen Vorschlag zu entwickeln, nach welcher Einarbeitungszeit der Grundsatz des Equal Pay zur Anwendung kommen solle. Im Frühjahr 2012 soll eine Kommission zur Findung einer Höchstabweichungsdauer von Equal Pay eingesetzt werden, sofern sich die Tarifvertragsparteien in der Branche bis dahin nicht auf einen gemeinsamen Vorschlag verständigen können.

\subsection{Tariftreuegesetze und vergabespezifische Mindestlöhne}

Auch im vergangenen Jahr hat sich die "Renaissance“ von Tariftreuegesetzen fortgesetzt. Die Zahl der Bundesländer, die die Vergabe öffentlicher Aufträge an die Einhaltung von Tarifstandards binden oder dies planen, ist auf mittlerweile elf gestiegen. In folgenden Bundesländern ist ein vergabespezifischer Mindestlohn vorgeschrieben:

- Berlin: 7,50 € (seit Juli 2010)

- Brandenburg: 8,00€ (seit Januar 2012)

- Bremen: 8,50€ (seit April 2011, vorher: 7,50 €)

- Rheinland-Pfalz: 8,50€ (seit März 2011)

- Nordrhein-Westfalen: 8,62€ (verabschiedet, in Kraft voraussichtlich in der ersten Jahreshälfte 2012).

Weitere Bundesländer mit Gesetzesinitiativen, die auch Mindestlöhne vorsehen, sind Baden-Württemberg (geplant 8,50€) und Hamburg (noch nicht festgelegt). MecklenburgVorpommern will laut Koalitionsvertrag einen Mindestlohn von 8,50 € einführen, Berlin will den Mindestlohn auf 8,50€ erhöhen. 


\subsection{Debatte um gesetzlichen Mindestlohn}

Ungeachtet der schrittweisen Ausweitung der branchenbezogenen Mindestlöhne nach dem AEntG ging die Debatte um einen allgemeinen, branchenübergreifenden Mindestlohn weiter. Vor allem in der CDU bemühten sich die Vertreter des Arbeitnehmerflügels unter dem ehemaligen NRW-Arbeitsminister Karl-Josef Laumann einen Vorschlag mehrheitsfähig zu machen, der einen gesetzlichen Mindestlohn auf Basis des tariflichen Mindestlohnes für die Leiharbeitsbranche vorsah. Laumann argumentierte, es handele sich dabei um einen von den Tarifparteien selbst ausgehandelten, also nicht staatlich gesetzten Mindestlohn, der bereits branchenübergreifend, nämlich in der Leiharbeitsbranche, zur Anwendung komme. Der Vorschlag fand zumindest in allgemeiner Form Unterstützung durch die Bundesarbeitsministerin, und auch die Bundeskanzlerin sprach sich für eine allgemeine Lohnuntergrenze aus. Eine parteiinterne Kommission sollte einen tragfähigen Vorschlag entwickeln, der auf dem Parteitag in Leipzig verabschiedet werden sollte. Der heftige Widerstand insbesondere seitens des CDU-Wirtschaftsflügels führte allerdings zu einer Verwässerung des Vorschlags. Der auf dem Parteitag gefasste Beschluss lautet: „Die CDU hält es für notwendig, eine allgemeine verbindliche Lohnuntergrenze in den Bereichen einzuführen, in denen ein tarifvertraglich festgelegter Lohn nicht existiert. Die Lohnuntergrenze wird durch eine Kommission der Tarifpartner festgelegt und soll sich an den für allgemein verbindlich erklärten tariflich vereinbarten Lohnuntergrenzen orientieren. Die Festlegung von Einzelheiten und weiteren Differenzierungen obliegt der Kommission. Wir wollen eine durch Tarifpartner bestimmte und damit marktwirtschaftlich organisierte Lohnuntergrenze und keinen politischen Mindestlohn." (CDU 2011)

Die Reaktion der Gewerkschaften war von Skepsis gekennzeichnet: Als keinesfalls großen Wurf, aber als Zeichen der Anerkennung gewisser Realitäten bezeichnete etwa Claus Matecki, DGB-Vorstandmitglied, den Beschluss. Es bleibe abzuwarten, ob es der CDU gelinge, diese „Mindestlohnlösung light" mit dem Koalitionspartner FDP umzusetzen. Inakzeptabel sei es, dass Lohnuntergrenzen nur dort von den Tarifpartnern vereinbart werden sollen, wo es nicht bereits Tarifverträge gibt. Das führe dazu, dass etwa viele Friseurinnen oder Floristen auch weiterhin Löhne von zum Teil unter $5 €$ erhielten.

Die sozialdemokratisch (mit)regierten Landesregierungen von Baden-Württemberg, Rheinland-Pfalz und Nordrhein-Westfalen brachten am 9. Dezember 2011 über den Bundesrat einen Entschließungsantrag ein, in dem die Bundesregierung aufgefordert wurde, unverzüglich einen Gesetzentwurf für einen allgemeinen flächendeckenden gesetzlichen Mindestlohn vorzulegen, der nicht unter 8,50€ liegen sollte (Bundesrat 2011).

\section{Ausblick}

In der Tarifrunde 2012 geht es in zahlreichen Tarifbereichen vorrangig um kräftige Entgelterhöhungen. Ähnlich wie in der vorangegangenen Tarifrunde gibt der vorlaufende Tarifabschluss in der Stahlindustrie vom November 2011 eine erste Orientierung. Nach dem Kündigungsterminkalender werden im ersten Halbjahr 2012 folgende größere Tarifbereiche das Tarifgeschehen bestimmen: der öffentliche Dienst (Bund, Gemeinden), dessen Verträge Ende Februar auslaufen, die Metall- und Elektroindustrie (März 2012), Volkswagen und chemische Industrie (Mai bis Juli 2012). Verhandlungen haben bereits in einigen Bereichen begonnen. Die bisher vorliegenden Forderungen bewegen sich zwischen 5 und 7 \%. Für die Deutsche Post AG wurde bereits ein erster Abschluss vereinbart: Er sieht eine Pauschale von $400 €$ und eine Tarifanhebung von 4,0 \% ab 1.4.2012 mit einer Laufzeit bis März 2013 vor.

Die Themen Regulierung der Leiharbeit und Übernahme der Ausgebildeten werden auch in der Tarifrunde 2012 eine Rolle spielen. In der Metallindustrie wurden

\section{ÜBERSICHT 4}

Bereits vereinbarte Tarifanhebungen für 2012/2013

\begin{tabular}{|c|c|c|c|}
\hline Tarifbereich & Erhöhung um & ab MM/2012 & bis MM/JJJJ \\
\hline Bauhauptgewerbe & $\begin{array}{l}2,3 \% \text { (West) } \\
2,9 \% \text { (Ost) }\end{array}$ & $\begin{array}{l}06 \\
08\end{array}$ & $03 / 2013$ \\
\hline Deutsche Bahn AG & $2,0 \%$ & 01 & $12 / 2012$ \\
\hline Druckindustrie & $\begin{array}{l}\mathbf{2 , 0} \% \\
150 € \text { zusätzliche } \\
\text { Einmalzahlung }\end{array}$ & $\begin{array}{l}08 \\
07\end{array}$ & $12 / 2013$ \\
\hline $\begin{array}{l}\text { Einzelhandel } \\
\text { Baden-Württemberg }\end{array}$ & $\begin{array}{l}2,0 \% \\
50 € \text { zusätzliche } \\
\text { Einmalzahlung }\end{array}$ & $\begin{array}{l}06 \\
04\end{array}$ & $03 / 2013$ \\
\hline $\begin{array}{l}\text { Groß- und Außenhandel } \\
\text { Baden-Württemberg }\end{array}$ & $2,4 \%$ & 05 & $03 / 2013$ \\
\hline $\begin{array}{l}\text { Hotels und Gaststätten } \\
\text { Baden-Württemberg }\end{array}$ & $2,4 \%$ & 07 & $06 / 2013$ \\
\hline Öffentlicher Dienst Länder & $1,9 \%$ plus $17 €$ & 01 & $12 / 2012$ \\
\hline Papier erzeugende Industrie & $1,6 \%$ & $01 / 2013$ & $05 / 2013$ \\
\hline Postbank AG & $2,4 \%$ & 04 & $04 / 2013$ \\
\hline $\begin{array}{l}\text { Privater Transport und } \\
\text { Verkehr NRW }\end{array}$ & $1,7 \%$ & 03 & $02 / 2013$ \\
\hline Süßwarenindustrie & $2,8 \%$ & $\begin{array}{l}\text { regional untersch. } \\
05-09 / 2012 \text { bzw. } \\
02 / 2013\end{array}$ & $\begin{array}{l}\text { regional untersch. } \\
04-08 / 2013 \text { bzw. } \\
01 / 2014\end{array}$ \\
\hline Textilreinigungsgewerbe & $2,3 \%$ & 06 & $05 / 2013$ \\
\hline Textilindustrie Ost & $2,3 \%$ & 04 & $03 / 2013$ \\
\hline Versicherungsgewerbe & $2,2 \%$ & 10 & $03 / 2013$ \\
\hline
\end{tabular}


dazu vor der eigentlichen Lohnrunde bereits Verhandlungen aufgenommen.

Das wirtschaftliche Umfeld der anstehenden Tarifrunde gestaltet sich dabei zunehmend schwierig: Die Prognosen sagen für Europa insgesamt eine Rezession und für Deutschland eine Stagnation der wirtschaftlichen Entwicklung voraus. Dabei mehren sich die Stimmen der Ökonomen (Wagner 2012; IMK 2012), die zur Stabilisierung der Binnennachfrage für eine Ausschöpfung des Verteilungsspielraums plädieren, was Tarifanhebungen von jahresdurchschnittlich 3,0 bis 3,5 \% bedeuten würde.

In einer Reihe von Tarifbereichen wurden bereits Tariferhöhungen für das Jahr 2012 und 2013 vereinbart. Die Abschlussraten bewegen sich zwischen 1,6 und $2,9 \%$ (Übersicht 4 ).

\section{LITERATUR}

Bispinck, R./WSI-Tarifarchiv (2005): Tarifpolitischer Jahresbericht 2004: Tarifstandards unter Druck, Informationen zurTarifpolitik, Januar, Düsseldorf Bispinck, R./WSI-Tarifarchiv (2010): Tarifpolitischer Jahresbericht 2009: Tarifpolitik in der Finanzmarktkrise, WSI-Informationen zurTarifpolitik, Januar, Düsseldorf

Bispinck, R. (2010): Niedriglöhne und der Flickenteppich von (unzureichenden) Mindestlöhnen in Deutschland, WSI-Report (4), Oktober

Bispinck, R./WSI-Tarifarchiv (2011): Tarifliche Vergütungsgruppen im Niedriglohnbereich 2011. Eine Untersuchung in 41 Wirtschaftszweigen, Elemente qualitativer Tarifpolitik (72), November, Düsseldorf

Bundesagentur für Arbeit (BA) (2012): Der Arbeits- und Ausbildungsmarkt in Deutschland, Monatsbericht Dezember und das Jahr 2011, Nürnberg Bundesinstitut für Berufsbildung (BiBB) (2012): Tarifliche Ausbildungsvergütungen 2011: Azubi-Gehälter legen deutlich zu, Pressemeldung vom 4.1., Bonn Bundesrat (2011) Drucksache 816/11 vom 9.12., Antrag der Länder Baden-Württemberg, Hamburg, Rheinland-Pfalz. Entschließung des Bundesrates - Faire und sichere Arbeitsbedingungen durch Implementierung eines flächendeckenden gesetzlichen Mindestlohnes, Berlin
Bundesvereinigung der Deutschen Arbeitgeberverbände (BDA) (2011): Geschäftsbericht 2011

Christlich Demokratische Union (CDU) (2011): Sonstige Beschlüsse des CDUParteitages vom 13.-15.11 in Leipzig, http://www.cdu.de/doc/pdfc/111115-sonstige-beschluesse.pdf (letzter Zugriff: 18.01.2012)

Institut für Makroökonomie und Konjunkturforschung (IMK), Arbeitskreis Konjunktur (2011): Im Bann der Austeritätspolitik. Prognose der wirtschaftlichen Entwicklung 2012, IMK Report (69), Dezember

Institut für Makroökonomie und Konjunkturforschung (IMK) (2012): Den Bann durchbrechen. Wirtschaftspolitische Herausforderungen 2012, IMK Report (70) Januar

Kalina,T./Weinkopf, C. (2010): Niedriglohnbeschäftigung 2008: Stagnation auf hohem Niveau - Lohnspektrum franst nach unten aus, Institut für Arbeit und Qualifikation, IAQ-Report 2010-06, Duisburg/Essen

Schulten, Th. (2011): Niedriglöhne in Deutschland, Ursachen, soziale Folgen und Alternativen, in: Wallraff. G./Bsirske, F./Möllenberg, F.J. (Hrsg.): Leben ohne Mindestlohn. Arm wegen Arbeit, Hamburg, S. 68-81

Wagner, G. (2012): Löhne wie aus dem Lehrbuch, DIW Wochenbericht $(1+2$ vom 4. Januar, Berlin

\section{AUTOREN}

REINHARD BISPINCK, Dr., ist Wissenschaftler im Wirtschafts- und Sozialwissenschaftlichen Institut (WSI) und Leiter des WSI-Tarifarchivs in der HansBöckler-Stiftung.

Reinhard-Bispinck@boeckler.de

GÖTZ BAUER, MONIKA MÜLLER, ULRICH SCHMIDT, MONIKA SCHWACKE-PILGER, ANDREA TAUBE und MONIKA WIEBEL sind Sachbearbeiterinnen und Sachbearbeiter im WSI-Tarifarchiv. 